Article

\title{
Study on the Effect of Flame Instability on the Flame Structural Characteristics of Hydrogen/Air Mixtures Based on the Fast Fourier Transform
}

\author{
Fu-Sheng Li, Guo-Xiu Li *, Yan-Huan Jiang, Hong-Meng Li and Zuo-Yu Sun \\ School of Mechanical, Electronic and Control Engineering, Beijing Jiaotong University, Beijing 100044, China; \\ 14116371@bjtu.edu.cn (F.-S.L.); 13121332@bjtu.edu.cn (Y.-H.J.); hongmengli@bjtu.edu.cn (H.-M.L.); \\ sunzy@bjtu.edu.cn (Z.-Y.S.) \\ * Correspondence: Li_guoxiu@yahoo.com; Tel./Fax: +86-10-5168-2047
}

Academic Editor: Chang Sik Lee

Received: 19 March 2017; Accepted: 10 May 2017; Published: 12 May 2017

\begin{abstract}
In this study, the effect of flame intrinsic instability on the flame structural characteristics of hydrogen/air mixtures premixed at various equivalence ratios were experimentally investigated from the macroscopic and microscopic perspectives, respectively. The correlation degree and the relative deformation degree were defined to quantitatively study the global flame structural characteristics. Peak detection was used to capture the characteristic length of the flame and fast Fourier transform was adopted to study the components of the fluctuation of the flame front. The results show that with the development of flames, the wrinkles in the flame front increase and the correlation degree of the flame decreases. The relative deformation degree of the flame first decreases and then increases. When the equivalence ratio is 0.6 , the average characteristic length initially exhibits an increasing trend, followed by a decreasing trend. The average characteristic length scale gradually increases, and the growth rate gradually decreases when the equivalence ratio ranges from 0.70 to 0.99 . With the increase in the wavenumber, the amplitude of the corresponding disturbance exhibited an increasing trend followed by a decreasing one. With the development of the flame, the maximum amplitude of the disturbance shows a reverse trend, i.e., first decreasing and then increasing. The disturbances with smaller wavelengths could be further developed.
\end{abstract}

Keywords: flame intrinsic instability; hydrogen/air; structural characteristics; equivalence ratio; fast Fourier transform

\section{Introduction}

The automotive industry is a pillar of the national economy, and it is the crystallization of human wisdom and a main driver of macroeconomic growth and stability, which significantly promotes the development of human civilization. Our automobiles, trains, and planes are fueled almost exclusively by petroleum products such as gasoline and diesel. However, the limited storage of fossil fuel and the increasing environmental crisis indicate that more attention should be paid to clean and renewable energy [1-4]. Development of cleaner alternative fuels and advanced power systems for vehicles has become a high priority for many governments and vehicle manufactures around the world. Hydrogen, with lower ignition energy and faster burning velocity compared to gasoline, acts as an ideal energy alternative. It can be produced from numerous materials, and has been recognized as the most promising alternative to fossil fuel $[5,6]$. So far, extensive research efforts have been devoted to the experimental investigation of the feasibility of using pure hydrogen as fuel in the internal combustion engine, and the results showed a significant increase in the thermal efficiency of the engine. Hydrogen was also studied as an additive for natural gas, diesel, and gasoline in the internal combustion engine [7-10]. 
The flame in the internal combustion engine with gasoline as fuel is an expanding flame; moreover, the research on laminar premixed combustion is of great significance to the understanding of combustion mechanism and the study of turbulent combustion [11,12]. Researchers [13-15] have experimentally studied the laminar burning velocity of hydrogen in the constant combustion bomb under different conditions. Furthermore, most previous studies on hydrogen/air premixed flame have focused on the intrinsic instability of premixed combustion [16,17]. Diffusive-thermal instability, resulting from the inequality of heat conduction and mass diffusion, and hydrodynamic instability, caused by the density ratio, are the two major factors that lead to a significant increase of flame surface area and flame propagation speed $[18,19]$. As a result of the existence of the flame intrinsic instability, the structural characteristics of the flame change dramatically. In other words, the surface of the flame exhibits the emergence of the cracks and irregular cellular structure. Further, the flame surface begins to become unstable and this results in the exponential growth of the flame radius over time, rather than monotonous growth [20]. For this reason, the effect of flame intrinsic instability attracts the attention of researchers, and it is always considered in combustion models [21].

In order to study the effect of the flame intrinsic instability on the flame structure, fractal geometry and statistical theory are important means of quantifying the cellular structure. The fractal dimension derived from fractal geometry is an important parameter to quantify the degree of folds of the flame profile [22,23] and is widely used in turbulent combustion models [24,25]. The quantified study on the cellular structure under the detonation condition were studied in [26,27], Moreover, Askari and Jiang also carried out quantitative studies on the cellular structure of the expanding flame [28,29]. In the linear stability theory, the perturbation of flame instability always imposes an initial flame kernel, and the flame amplitude evolves exponentially [30,31]. Moreover, several researchers have also studied the dispersion relation for the flat flame [32]. In the linear stability theory, in the dispersion equation and the M-S equation, the application of Fourier transform (FT) is of great significance. Fourier theory shows that any continuous measurement of the timing or signal can be expressed as a different frequency sine wave signal infinite superposition. Due to its important role, Fourier transform is widely used in scientific research. Askari [33] uses fast Fourier transform (FFT) to analyze the pressure in the combustion chamber and the combustion instability of synthetic natural gases. The results of Kawahara show that the pressure wave would damage the engine cylinder wall during knocking in a hydrogen spark-ignition engine by using FFT [34]. In addition, the FFT is used in other fields [35-37]. For the wrinkled flame front, the perturbation of the flame front is also a perturbation signal, which can reflect the distribution and development of the frontal perturbation from the microscopic point of view. For this reason, a further study is necessary.

The existing experimental research on the spherical expansion flame basically use the schlieren and high-speed photography technology to capture the date of the flame evolution. Researchers focuses on the phenomenology to quantifiably study the flame structural characteristics. However, for expansion during the process of data collection, the flame will actually be reduced dimension processing. That is, the flame images obtained by the schlieren are two-dimensional images, resulting in a large amount of information loss, such as the true value of the cracks and the area of cells on the flame surface distortion. However, the flame contours are not disturbed. Further analysis of the flame contours is an important way to reveal the mechanism of the effect of flame intrinsic instability on the structural characteristics of the flame.

In this study, a series of experiments were performed on a mixture of hydrogen/air with equivalence ratios ranging from 0.6 to 0.99 under an initial pressure of $0.1 \mathrm{MPa}$ and an initial temperature of $300 \mathrm{~K}$ in a constant volume combustion bomb. The correlation degree and relative deformation degree were defined to quantitatively study the change of flame front from the macroscopic perspective. Peak detection and fast Fourier transform (FFT) were used to study the structural characteristics from the microscopic perspective. Moreover, the structure of flame front in the near ignition electrodes region was also systematically analyzed. 


\section{Experiment Setup and Procedures}

All of the experiments were conducted in a constant volume combustion bomb (Beijing Anshi Tong Technology Co., Ltd., Beijing, China) with an inner diameter of $140 \mathrm{~mm}$. Horizontally opposed quartz windows (Beijing Anshi Tong Technology Co., Ltd., Beijing, China) of $100 \mathrm{~mm}$ offered the optical access. The mixture was ignited by the electrodes located horizontally in the center of the bomb. The mixture flame images were obtained by using an FASTCAME SA-X2 high-speed digital camera (Fastcam, Tokyo, Japan), with a sampling frequency of 13,500 per second. The component gases controlled by the pressure gauge were supplied into the chamber through an inlet valve according to the calculated partial pressure. To ensure the uniformity of the mixture, ignition was activated $5 \mathrm{~min}$ after placing the mixture in the chamber. The combustion products were taken out after complete combustion by using a vacuum pump, and a nitrogen flow was used to purge the constant combustion chamber in order to eliminate the impact of residual gas on the experimental results. A schematic illustration of the experimental setup is shown in Figure 1.

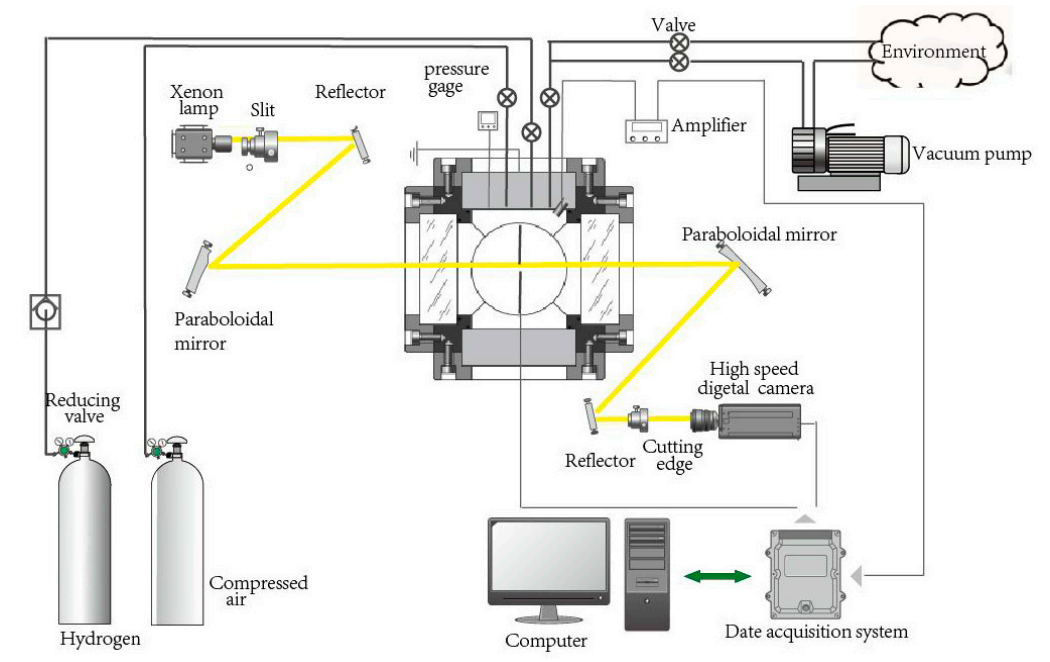

Figure 1. Schematic illustration of the experimental setup.

The structure of the premixed spherical flame contains rich information. Extraction of the information regarding the flame structure geometry not only leads to the deepening of the comprehensive understanding of the premixed combustion mechanism, but also provides important experimental data for the improvement of the combustion model. In addition to the numerous flame images, MATLAB was selected to obtain the information about the flame. Figure 2 shows the comparison between the original flame and the extraction edge and Figure 3 exhibits the edges of the evolution flame. The results show that the flame extracted by using the program displayed a higher coincidence with the flame profile, which laid a foundation for the follow-up study.

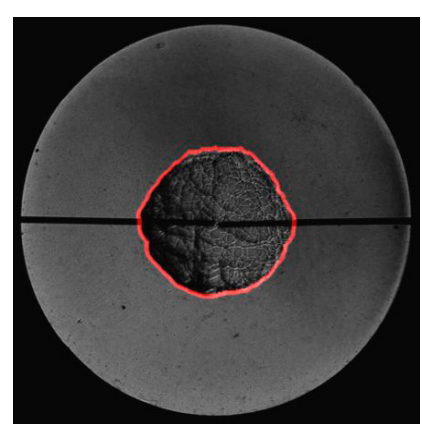

Figure 2. The comparison between the original flame and extraction edge. 


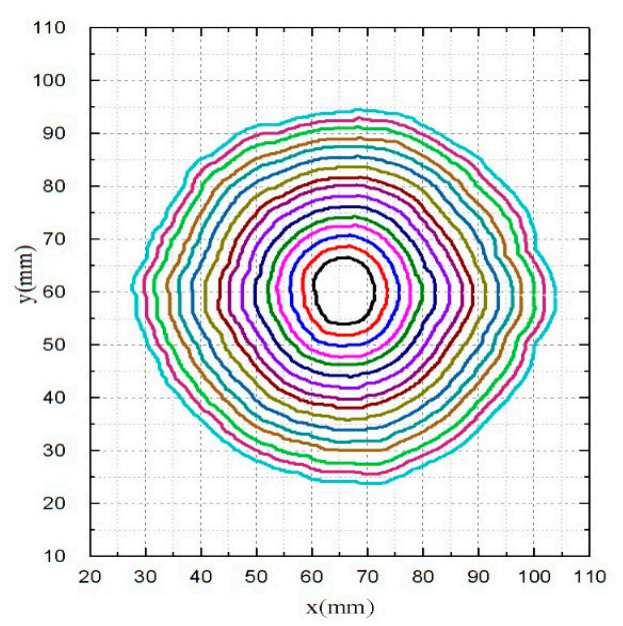

Figure 3. The extraction edges of flame.

For the expansion spherical flame, polar coordinates is the most suitable coordinate system for statistical flame profile information. The centroid of the flame image was obtained by MATLAB, and the coordinate system was established based on the coordinate center. $\theta$ represents the angle from $\mathrm{OA}$ to $\mathrm{OB}$. In order to obtain more detailed information about the flame front, starting from $\theta=0^{\circ}$, the coordinate information of the flame front was stored every $0.004^{\circ}$. Moreover, three radii were defined to quantify the deformed flame under the effect of flame instability, namely, $R_{r}$ representing the radius of the flammable area, $R_{t}$ representing the outside area with fresh mixture, and $R_{a}$, indicating the flame radius equivalent to the flame area (Figure 4). For the process of outwardly propagating spherical flame, the flame front does not show a standard circle under the interaction of external factors such as ignition energy, electrodes, and the internal instability of the flame, i.e., the flame intrinsic instability. The flame front is an irregular circle between $R_{r}$ and $R_{t}$. The local flame radius $\left(R_{i}\right)$ on the flame front can be considered the sum of the mean flame radius $R_{a}$ and the fluctuation radius $R_{i}^{\prime}$ as follows:

$$
R_{i}=R_{a}+R_{i}^{\prime}
$$

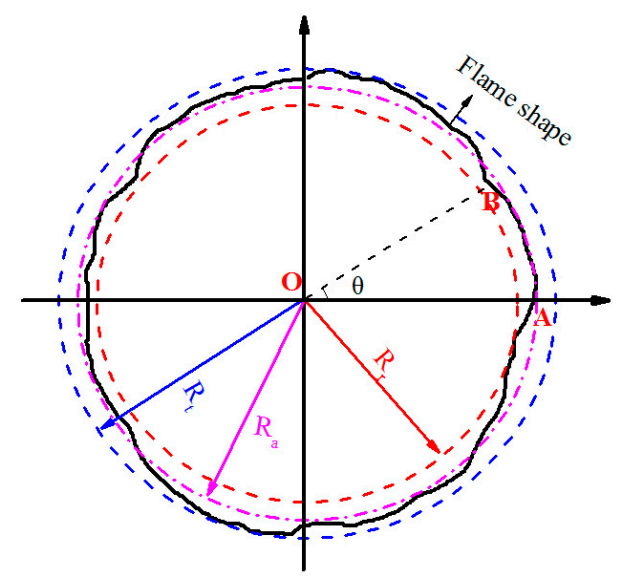

Figure 4. Schematic illustration of the related parameter definition of flame front.

During the evolution of the flame front, the flame front changes from a stable state to an unstable one, followed by the gradual appearance of cracks, and finally to the self-turbulence state. There must be some relevance between the flame fronts at different times (Figure 3). To study the correlation of the flame under the influence of flame instability, the correlation degree (Equation (2)) of the flame front at 
different time $\left(t_{i}\right)$ was analyzed in the process of flame evolution. The lower value indicated a more unstable flame front.

$$
\rho_{R_{a m}, R_{a n}}=\sum_{i=1}^{N}\left(R_{m i}-R_{a m}\right)\left(R_{n i}-R_{a n}\right) / \sqrt{\sum_{i=1}^{N}\left(R_{m i}-R_{a m}\right)^{2}} \sqrt{\sum_{i=1}^{N}\left(R_{n i}-R_{a n}\right)^{2}}
$$

While $R_{m i}$ and $R_{n i}$ represent the local radii of the flame at different times, $R_{a m}$ and $R_{a n}$ are the flame radii equivalent to the flame area at different times. The correlation degree ranges from -1 to 1 . When the correlation degree turns to 1, the shapes of the flame front at different times are similar. With the decrease of the correlation degree, the difference of the flame shape at different times increases. When the correlation degree turns to -1 , the shape of the flame front at different time are completely opposite. To quantify the wrinkles in the flame front, relative deformation degree is defined as follows:

$$
\xi=\sum_{i=1}^{N}\left(R_{m i}-R_{a m}\right)^{2} / \sum_{j=1}^{N}\left(R_{n j}-R_{a n}\right)^{2}
$$

According to the literature [38], when the flame radius is greater than $6 \mathrm{~mm}$, the flame propagation speed is no longer affected by the ignition energy. Moreover, to study the change of self-similar flame from the stable flame propagation process to the unstable flame propagation process (in which flame front shows the appearance of cracks or cellular structures), while avoiding the effect of pressure on the flame propagation, the scope of the flame radius was selected from 8 to $22 \mathrm{~mm}$.

Under the influence of the flame intrinsic instability, the flame front shows a gradual appearance of cracks followed by the development of cellular structure. The flame front no longer keeps the standard circular shape, due to the varying degrees of disturbances applied to the flame surface. In fact, the fluctuation in the flame front is a form of energy, which reflects the degree of flame instability. To study the fluctuation of the flame front, discrete Fourier transform (DFT) was used. Let $\left(x_{i}\right)$ be a sequence of length $N$, then its DFT is the sequence $\left(F_{n}\right)$ represented as follows:

$$
F_{n}=\sum_{i=0}^{N-1} x_{i} e^{-\frac{2 \pi j}{N} n i}
$$

For FFT to be highly efficient, the computational complexity was reduced from $\mathrm{O}\left(n^{2}\right)$ to $\mathrm{O}$ $(n \cdot \log \cdot n)$, and was used in the subsequent analysis. In order to accurately measure the amplitudes and the phase of the frequency, leakage should be taken into consideration (leakage is caused by the FFT's assumption that the input signal repeats periodically and that the periodic length is equal to the length of the actual input). Window functions, including Triangular, Bartlett, Welch, Hann, Hamming, and Blackman, are good choices to mitigate leakage in the measured FFT. In this study, Hann window [39-41] was selected.

\section{Results and Discussion}

For the outwardly propagating laminar premixed flame, the flame instability plays an important role in the development of the flame dynamics, causing wrinkles in the flame surface and leading to changes in the combustion flow field. Therefore, the laminar flame gets gradually transformed into an unstable state and eventually develops into a turbulence flame. Hydrogen/air mixtures are very susceptible to thermal-diffusive instability due to the high molecular diffusivity of hydrogen. The thermal-diffusive instability can cause local changes in mixture composition and reaction rate, and subsequently flame front wrinkling. The wrinkling effects eventually contribute to augmentation of the burning velocity. Figure 5 shows the evolution of flame front of hydrogen/air mixture under different equivalence ratios. When the equivalence ratio is less than one, the thermal-diffusive instability plays a dominating role. Smaller equivalence ratio results in stronger thermal-diffusive 
instability [16]. In the early stage of the flame development, the flame surface is smooth. With the development of the flame, cracks gradually emerge on the surface of the flame, which then develop into a cellular structure under the flame instability. With the increase of the equivalence ratio, the flame radius of the emerging cracks increases, the number of cells on the flame surface decreases, and the area of the cells increases. Moreover, the flame counter maintains a certain degree of correlation in the flame development process. Therefore, it is necessary to carry out the relevant research to reveal the effects of flame intrinsic instability on the flame structure.

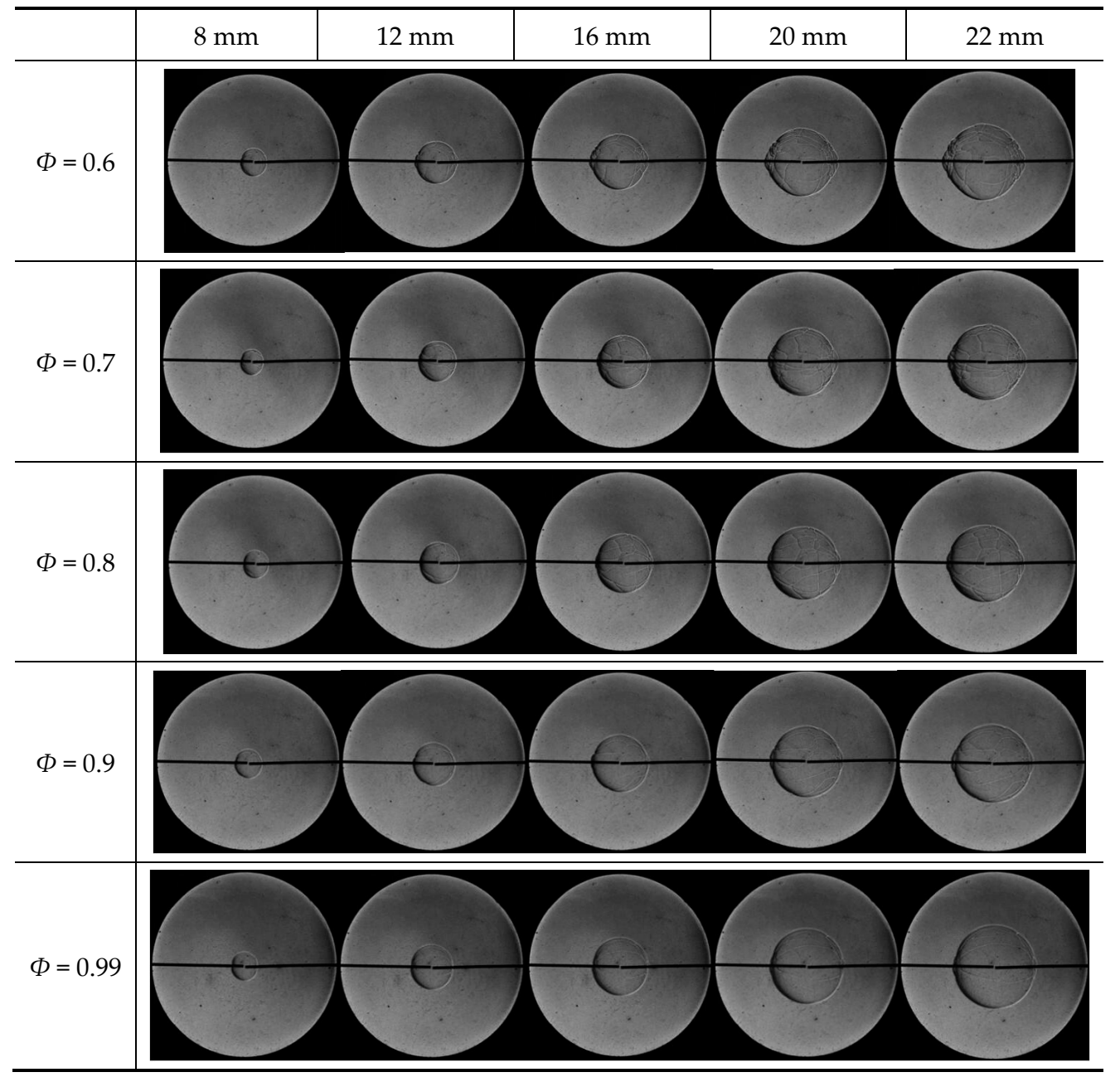

Figure 5. Evolution of flame front of hydrogen/air mixture under different equivalence ratios.

In order to study the development of flame front intuitively, Figure 6 uses the polar coordinates to expand the extracted flame profile. For the influence of the flame intrinsic instability, the flames gradually exhibit different degrees of wrinkles and even progress to self-turbulence. The flame surface is no longer smooth. As can be seen by polar coordinates, the expansion of the flame profile is no longer a smooth line, and the entire line receives varying degrees of bending, and it becomes irregular. As a whole, with the development of the flame, the degree of irregularity of the flame front increases. In terms of the local development of the flames, there are two typical development modes. With the development of the flame, the degree of folds of the flame surface gradually increases in Region 1 and Region 2. The flames of the smaller wrinkle gradually weakened or even disappeared with the development of the flame in Region 3. The local flame radius in Region 1 and Region 2 are very similar in shape and amplitude. The flame shape shows a symmetrical trend, but the local details of the flame are very different. This may be a result of the development of the disturbances in the flame under the 
limit of the ignition electrodes. These two completely different trends in the development of flame wrinkles are affected by the impact of flame stretch rate. Later, the effects of stretch rate on the whole flame and the local wrinkle from the micro and macro point of view were studied.

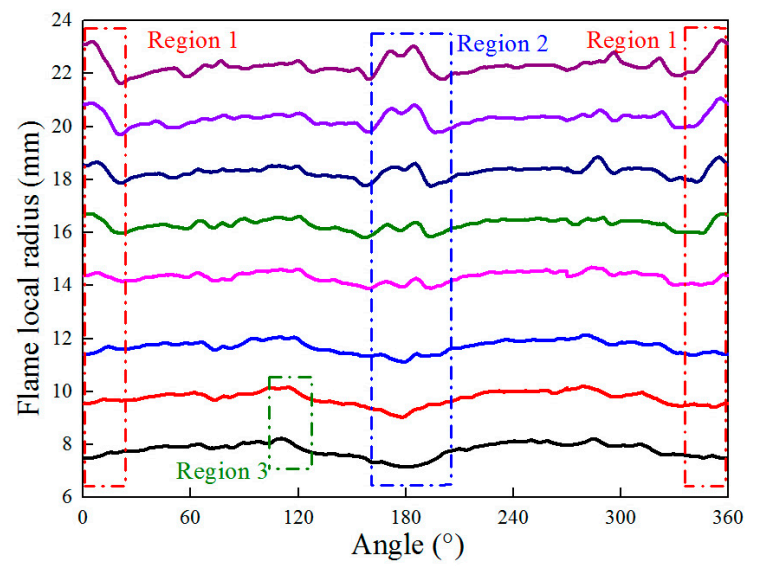

Figure 6. The polar coordinates of flame development under equivalence ratio of 0.8 .

Figure 7 shows the evolution of flame correlation degree under different equivalence ratios. Clearly, with the development of the flame, the correlation degree of the flame front is gradually reduced, even to below 0 . When the equivalence ratio is 0.60 , the flame correlation degree decreases rapidly with the development of the flame and reaches zero at the flame radius of $13.6 \mathrm{~mm}$. When the equivalence ratio is 0.99 , the flame correlation degree changes only slightly at the beginning of the flame propagation until the flame radius becomes greater than $14 \mathrm{~mm}$. The value reaches the minimum $(0.36)$ at the flame radius of $22 \mathrm{~mm}$. When the equivalence ratios are in the range of 0.7 to 0.8 , the evolution of flame correlation degree is similar and the flame correlation degree becomes negative in the later stage of the flame development. With the development of the flame, the small-scale structures in the flame surface gradually increase with the development of the flame, and the interference and merge between small-scale structures directly affects the flame correlation degree, leading to a decrease in the value. With the increase of the equivalence ratio, the thermal-diffusive instability of the flame gradually weakens and the wrinkles in the flame front decrease, leading to the occurrence of an increasing trend for the correlation degree.

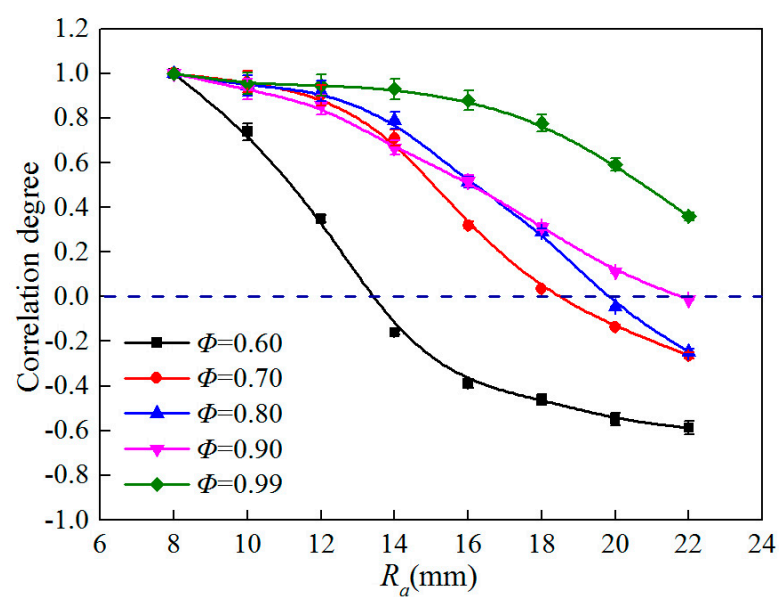

Figure 7. The evolution of flame correlation degree under different equivalence ratios.

With the development of the flame, the flame front no longer retains a smooth state. Under the influence of both large-scale structures and small-scale structures on the flame surface, the flame front 
forms a different degree of folds. Figure 8 demonstrates that the flame global deformation degree, compared to the value when the average flame radius is $8 \mathrm{~mm}$, first shows a decreasing trend and then an increasing trend with the development of the flame. The radius corresponding to the minimum relative deformation degree gradually increases with the increase of the equivalence ratio. In the early stage of the flame evolution, the shape of the ignition spark has a significant effect on the flame shape. Moreover, the ignition electrodes also affect the flame shape at the same time, so that the flame is no longer circular shaped at the beginning of flame evolution. With the evolution of the flame, the stretch rate of the flame decreases gradually, leading to the limitation of the flame initial shape reduce. Moreover, the emergence of the cellular structures in the flame front, caused by the flame intrinsic instability, further offset the influence of the shape of the spark, resulting in a decreasing trend followed by an increasing trend for the relative deformation degree of the flame. The smaller the equivalence ratio, the stronger thermal diffusive instability. The complex cellular structure on the flame surface results from complex large-scale and small-scale structures on the flame front, allowing the flame to counteract the influence of the initial flame shape at a small radius, i.e., to a minimum value. With the further development of the cellular structure in the flame front, the relative deformation degree of the flame gradually increases. When the equivalence ratio is 0.99 , the thermal-diffusive instability of the flame is the weakest, and the number of cracks and cells on the flame surface is the lowest.

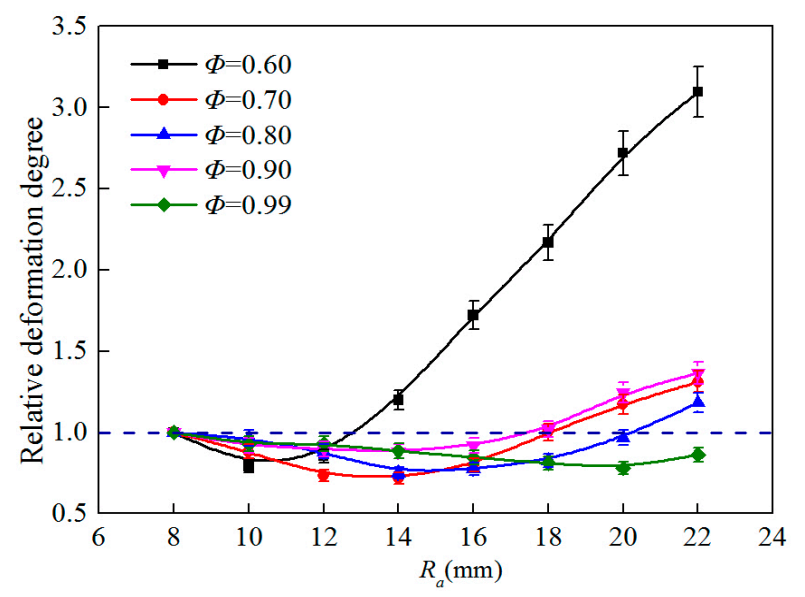

Figure 8. The relative deformation degree of flame evolution under different equivalence ratios.

Figure 9 displays the evolution of the characteristic length scale in the flame front under different equivalence ratios. The wrinkles of the flame surface are actually due to the superposition of different scales of disturbances, the larger disturbances are less affected by the flame stretch rate and develop with the evolution of the flame. The smaller scale disturbances are influenced by the local stretch rate and the interaction between the disturbances, showing the disappearance and growth of one after another. The points on the flame front represent the minimum value of the concaves on the flame surface. Clearly, there are two types of points in the evolution of the flame along the radial direction. One continuously appears and the other suddenly disappears or abruptly occurs. The arc length between the points that develops along the radial direction of the flame is larger and continues to increase with the development of the flame. The arc length between the adjacent two points, which is defined as characteristic length in the subsequent investigation, first increases and then decreases. This indicates that the longer wavelength disturbances always affect the flame front and the shorter wavelength disturbances are enhanced or weakened due to the interaction among different disturbances during the development of the flame. Moreover, the boundary point of the longer wavelength disturbances undergoes a slight shift under the influence of smaller wavelength disturbances. When the equivalence ratio increases, the number of points captured by the flame surface 
decreases. This indicates that the degree of deformation of the flame surface decreases and the surface of the flame tends to be smoother.

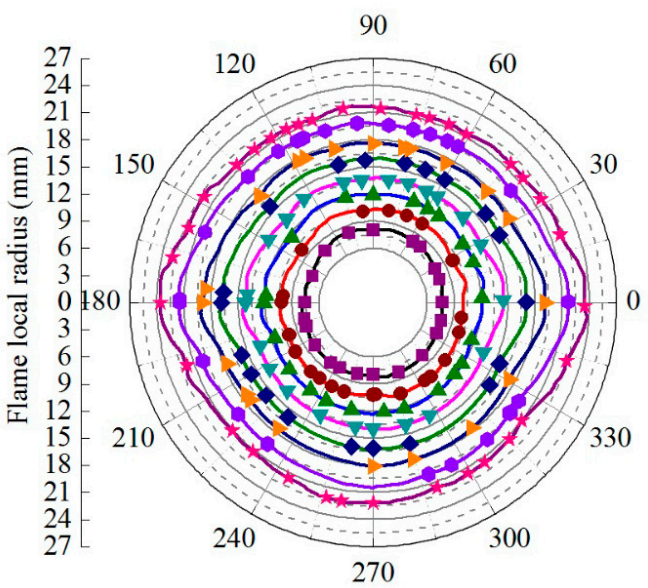

(a) $\Phi=0.6$

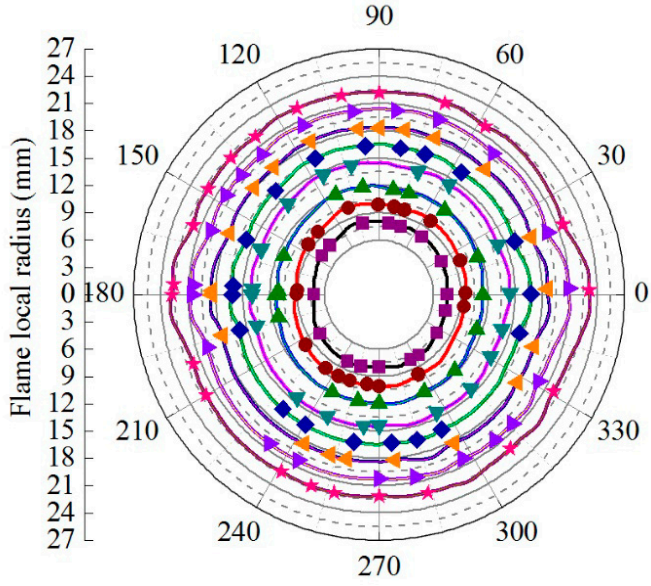

(c) $\Phi=0.8$

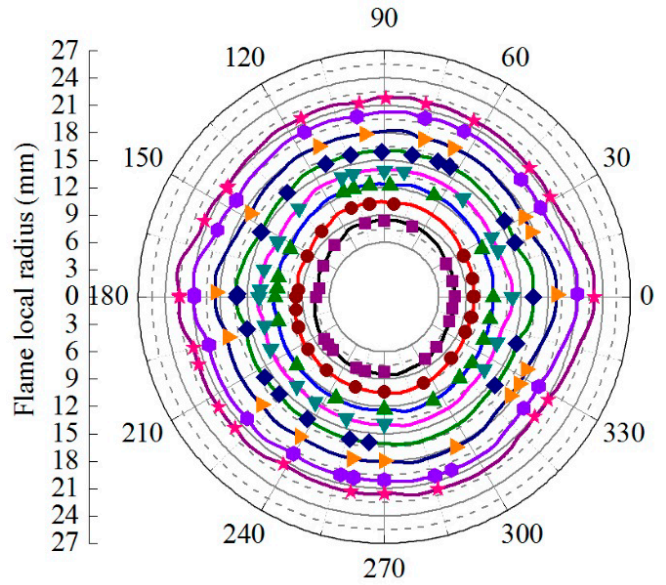

(b) $\Phi=0.7$

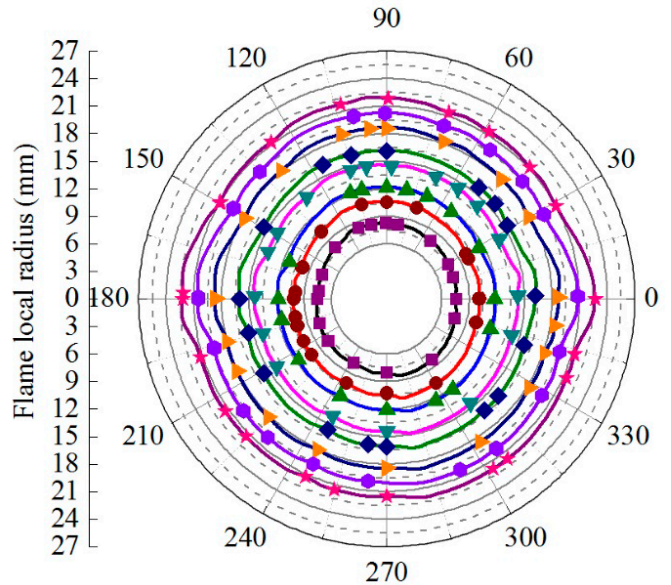

(d) $\Phi=0.9$

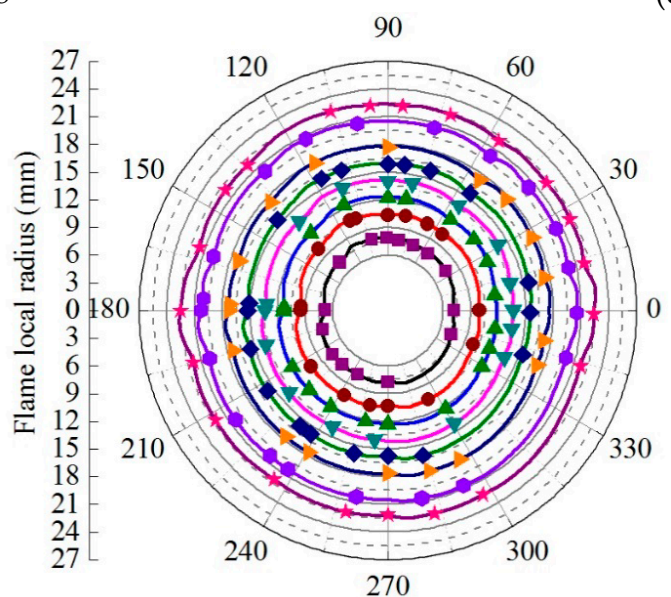

(e) $\Phi=0.99$

Figure 9. The evolution of the characteristic length scale in the flame front under different equivalence ratios.

To further study the variation of the characteristic length of the flame surface, the average characteristic length of the flame surface was calculated under different flame radii. Under the lower equivalence ratio (0.6), the average characteristic length presents first an increase and then a decrease 
tendency. When the equivalence ratio was higher than 0.6, the average characteristic length scale gradually increased, and the growth rate gradually decreased. The average characteristic length shows an increasing trend with the increase of the equivalence ratio (Figure 10). This is mainly attributed to the large stretch of the flame in the early stage of flame development restricting the development of cellular structures in the flame front, thus resulting in an increase in the average characteristic length in the flame surface. With the development of the flame, the stretch in the flame gradually decreases and the flame instability is further developed. The cellular structures in the flame surface develop faster than the expansion of the flame, leading to a decrease in the growth rate of the average characteristic length.

In order to further study the fluctuation in the flame surface, the FFT was performed to investigate the fluctuation in the flame front. The disturbances in the flame surface can be divided into three parts according to the distribution of the disturbances. When the wavenumber ranges from 0 to 10 , the disturbances are the major factor of the flame structure. Combined with the characteristic length of the flame surface, the wavenumber of the disturbances directly affecting the cellular structure in the flame surface can be calculated in the range of 10 to 280 . However, the disturbances which wavenumber larger than 280 is considered an interference signal. Therefore, the disturbances that have wavenumbers ranging from 0 to 10 and 10 to 280 were studied.

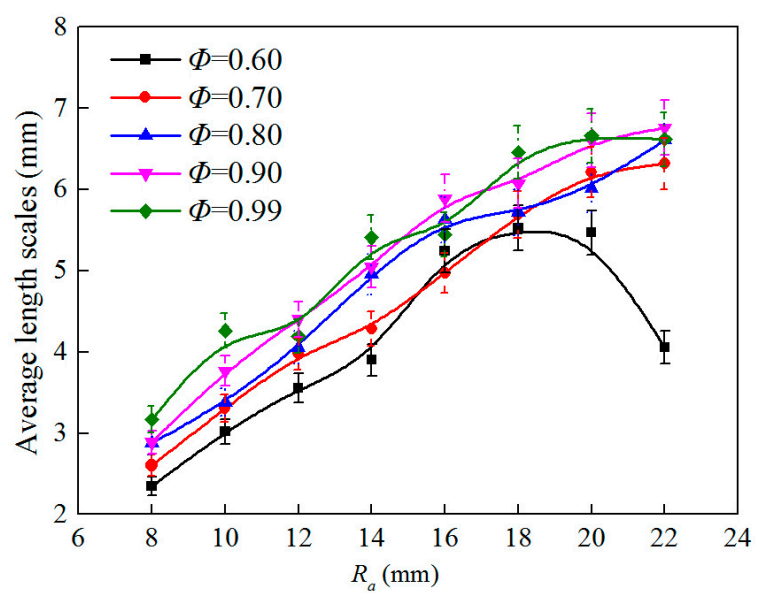

Figure 10. The evolution of the average length scale in the flame front under different equivalence ratios.

Figure 11 shows that with the increase of the wavenumber, the amplitudes of the corresponding disturbances present a tendency to first increase and then decrease, the fluctuation in amplitude also shows a similar trend. The larger amplitudes of the disturbances are mainly concentrated on wavenumbers ranging from 0 to 2 and the amplitudes of the disturbances corresponding to the different wavenumbers are quite disorganized. When the wavenumber ranges from 1 to 2 , the amplitudes of the disturbances present an increase tendency with the development of the flame. However, when the wavenumber ranges from 2 to 10, the amplitudes of disturbances are relatively small. This is mainly attributed to the fact that the flame stretch significantly affects the development of cellular structure in the flame at the beginning of the flame evolution. The larger initial stretch limits the development of the smaller wavelength disturbances, and only the larger wavelength disturbances affect the flame. With the further decrease of the stretch, the smaller wavelength disturbances are able to develop, which is the main reason for the appearance and strengthening of the cellular structures on the flame surface. With the increase of the equivalence ratio, the maximum value of the amplitude corresponding to the disturbance at the same radius shows a decreasing tendency, which also reflects the gradual increase in the smoothness of the flame surface with the increase of the equivalence ratios. 


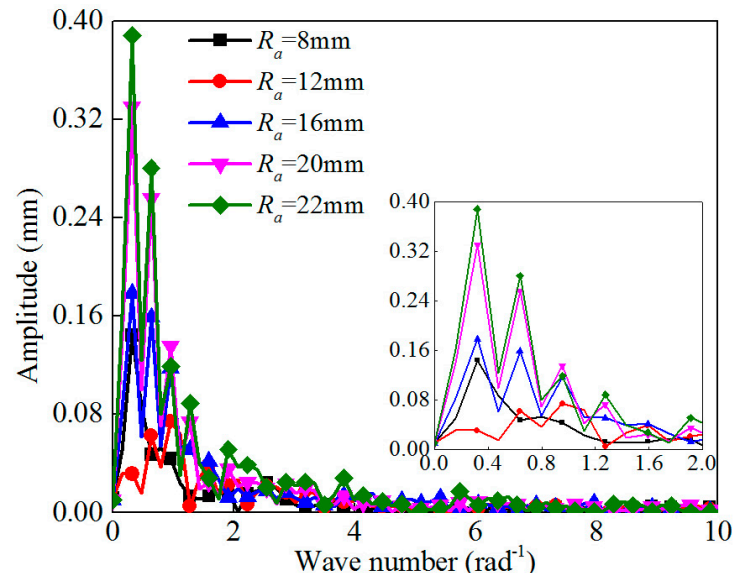

(a) $\Phi=0.6$

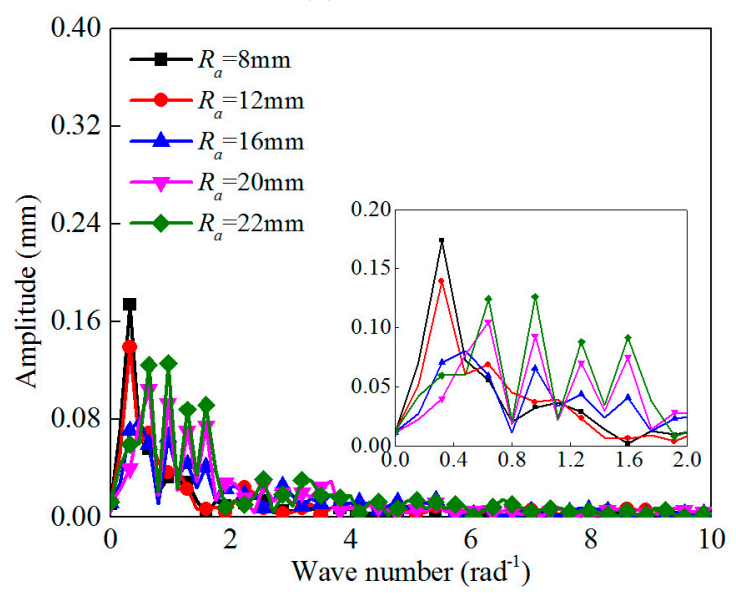

(c) $\Phi=0.8$

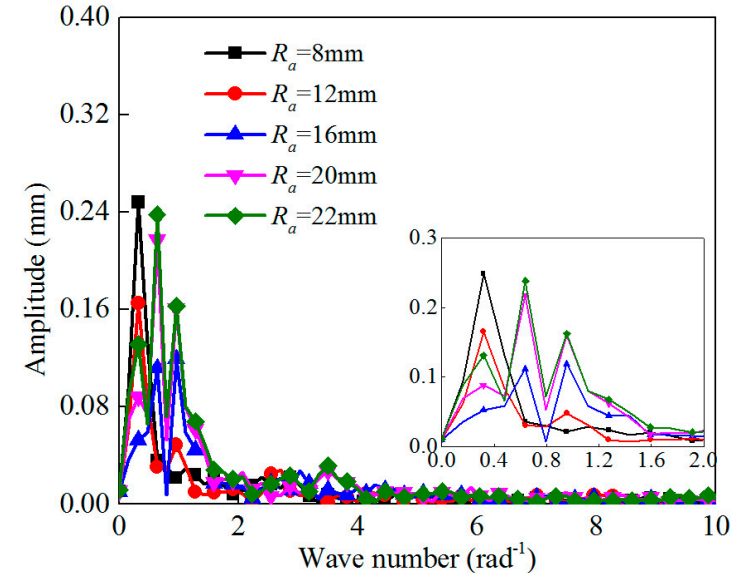

(b) $\Phi=0.7$

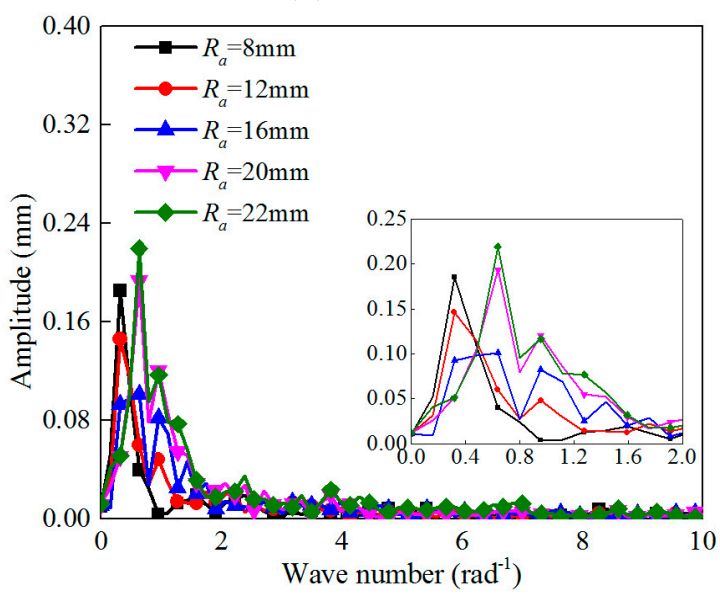

(d) $\Phi=0.9$

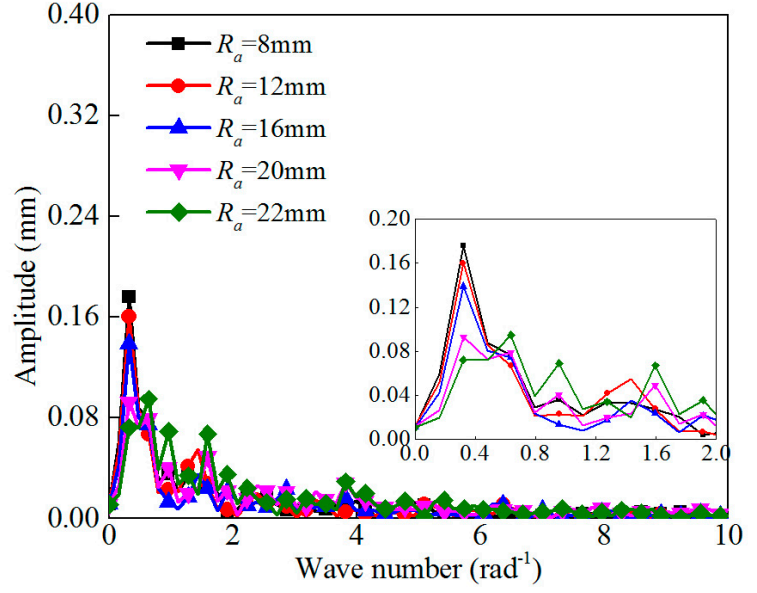

(e) $\Phi=0.99$

Figure 11. The distribution of disturbance disturbances range from 0 to 10 under different equivalence ratios.

Figure 12 demonstrates that with the development of the flame, the maximum amplitude of the disturbances first shows a decreasing trend and then an increasing trend. With the increase of the equivalence ratio, the radius corresponding to the maximum amplitude of the disturbance gradually increases in the early stage of the flame development. When the equivalence ratio is 0.60 , the maximum amplitude of the disturbances reaches to the minimum value at $12 \mathrm{~mm}$ and then increases rapidly. The maximum amplitude of the disturbances corresponding to the flame radius of $22 \mathrm{~mm}$ is 2.69 times 
that of $8 \mathrm{~mm}$. The maximum amplitude of the disturbances at the radius of $22 \mathrm{~mm}$ is 0.54 times that of the radius of $8 \mathrm{~mm}$ under the equivalence ratio of 0.99 . This shows that the development of smaller disturbances plays an important role in the wrinkle of the flame surface.

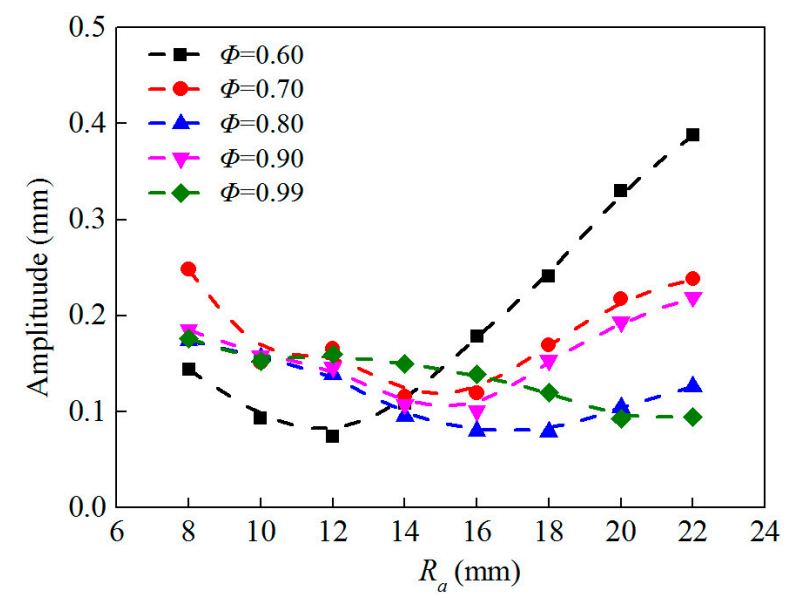

Figure 12. The maximum amplitude of the disturbances under different equivalence ratios.

Furthermore, the wavenumber corresponding to the maximum amplitude of the disturbances remains unchanged at the initial stage of flame development. With the development of the flame, the variation in the wavenumber gradually differs. Moreover, with the increase of the equivalence ratio, the radius corresponding to the wavenumber of the maximum amplitude begins to increase gradually (Figure 13). This is a good illustration that there is a strong interaction of different disturbances in the development of the flame resulting in the wavenumber corresponding to the maximum amplitude fluctuation.

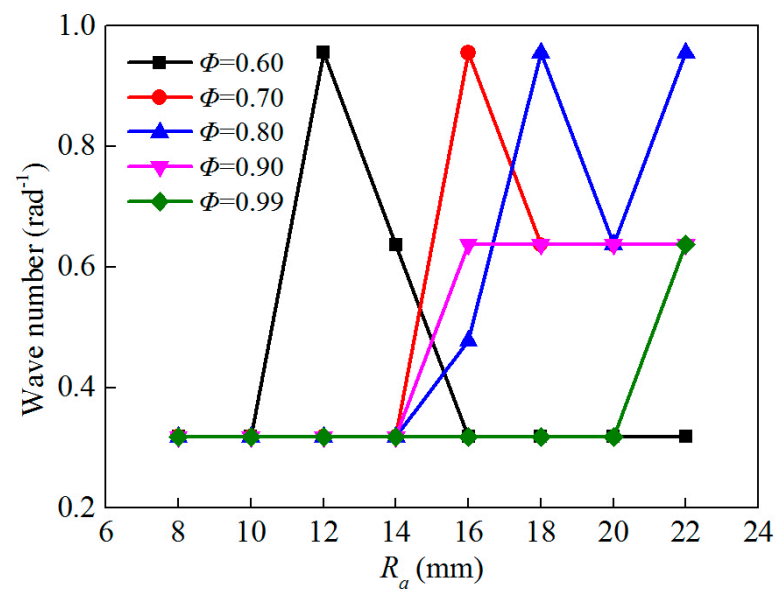

Figure 13. The wavenumber of the maximum amplitude under different equivalence ratios.

In order to study the variation of the disturbances in the zone for which the wavenumber ranges from 10 to 280, the amplitudes of the disturbances in this section were logarithmically processed and nonlinear fitting was adopted. Figure 14 demonstrates that the logarithm of the amplitude decreases gradually with the increase of wavenumbers, and the absolute value of the amplitude growth rate also decreases gradually. With the development of the flame, the amplitude of the disturbance of the same wavenumber increases. This indicates that with the development of the flame, the instability of the flame surface is enhanced and the disturbances with smaller wavelengths can be further developed, which results in the formation of the complicated cellular structures of flame surface. 


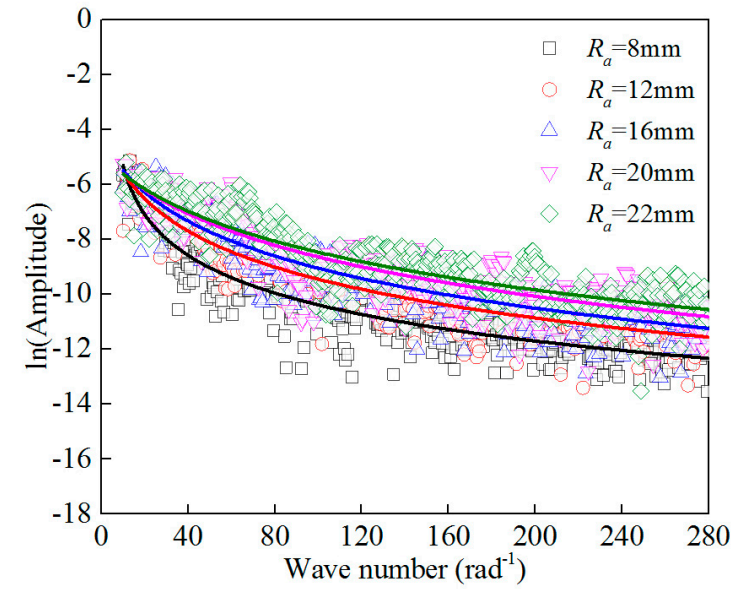

(a) $\Phi=0.60$

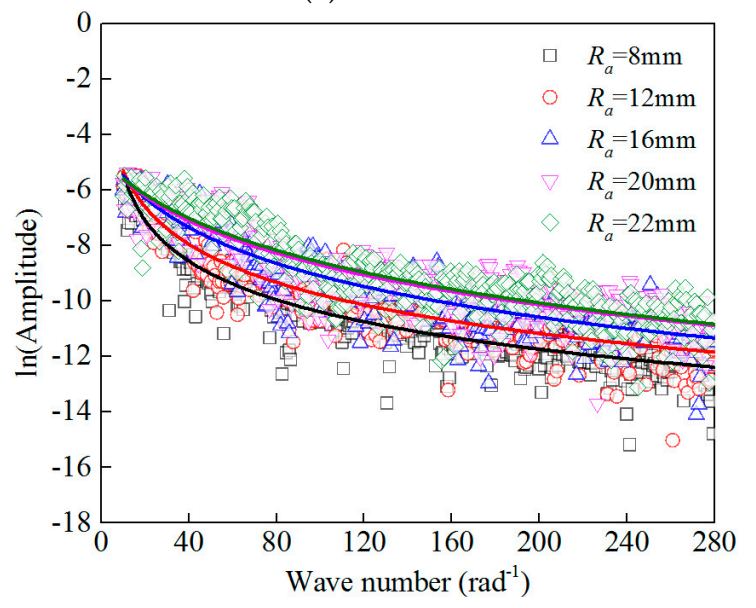

(c) $\Phi=0.80$

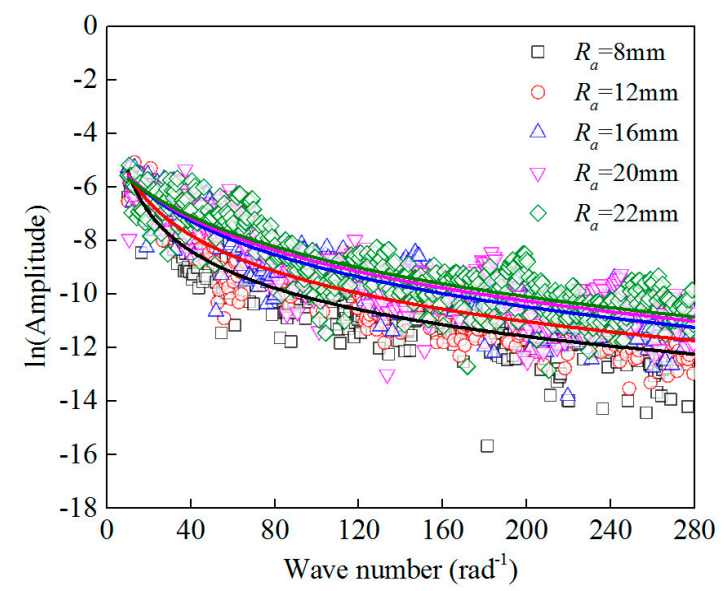

(b) $\Phi=0.70$

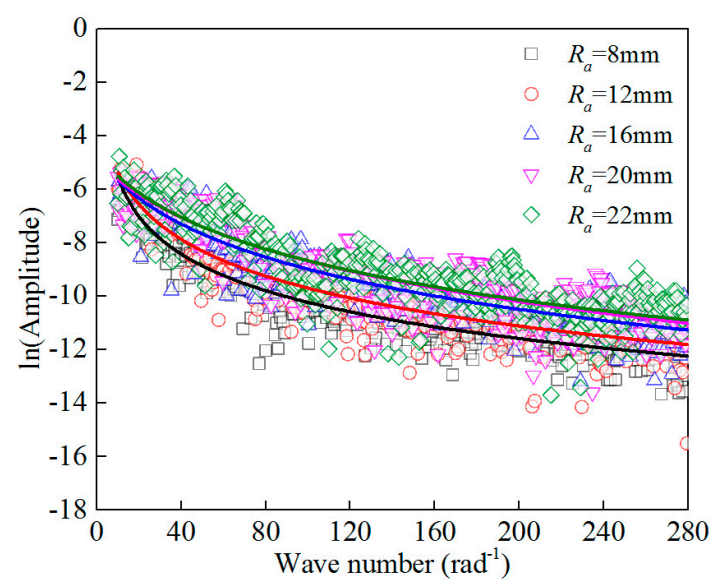

(d) $\Phi=0.90$

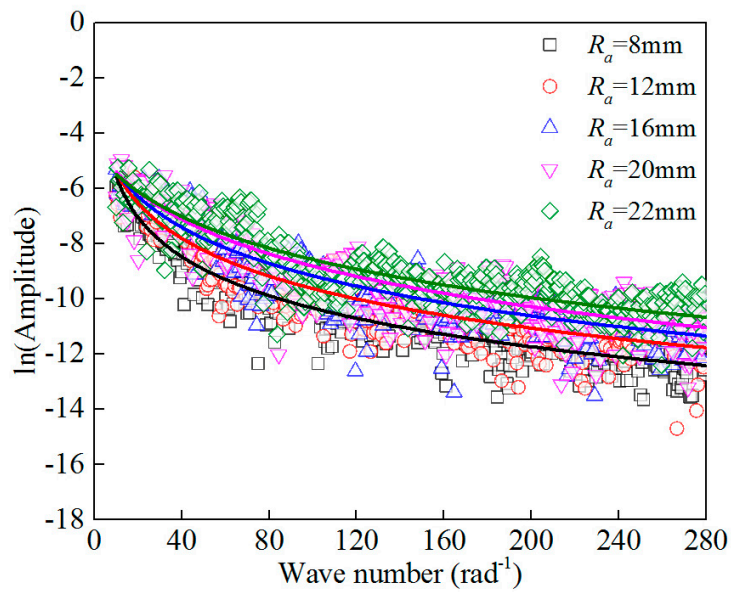

(e) $\Phi=0.99$

Figure 14. The distribution of disturbances range from 10 to 280 under different equivalence ratios.

\section{Conclusions}

In this study, the effect of flame intrinsic instability on the flame structural characteristics of hydrogen/air mixture were investigated under atmospheric pressure and temperature. The correlation degree and relative deformation degree were defined in order to study the evolution of the global flame structural characteristics. Peak detection and FFT were used to study the structural characteristics from the microscopic perspective. The main conclusions can be summarized as follows: 
- With the development of the flame, the surface of the flame shows that cracks gradually appear and then develop into cellular structures under the effect of the flame intrinsic instability. With the increase of the equivalence ratio, the effect of the thermal-diffusive instability on the flame front decreases, and the cellular structure in the flame surface gradually weakens.

- With the development of the flame, the correlation degree of the flame front gradually decreases, even to below 0 . The flame global deformation degree relative to the values when the average flame radius is $8 \mathrm{~mm}$ shows a decreasing trend followed by an increasing trend. With the increase of the equivalence ratio, the thermal-diffusive instability of the flame gradually weakens and the wrinkles in the flame front decrease, leading to an increasing trend of correlation degree.

- There are different scale disturbances in flame front which result in the formation of different characteristic length scales. Under the lower equivalence ratio (0.6), the average characteristic length presents first an increase tendency and then a decrease tendency. When equivalence ratio is higher than 0.6, the average characteristic length scale gradually increases, and the growth rate gradually decreases. The average characteristic length shows an increasing trend with the increase of the equivalence ratio.

- With the increase of the wavenumber, the amplitudes of the corresponding disturbances show an increasing trend first, followed by a decreasing trend. The larger amplitudes of the disturbances were mainly concentrated in wavenumbers ranging from 0 to 2 . When the wavenumber ranges from 1 to 2 , the amplitudes of the disturbances present an increase tendency with the development of the flame. However, when the wavenumber ranges from 2 to 10, the disturbance amplitude is relatively small. The evolution of the flame front is the result of the interaction between the disturbances and the stretch rate.

- When the wavenumber ranges from 10 to 280 , the logarithm of the amplitudes decreases gradually with the increase of wavenumbers, and the absolute value of the amplitude growth rate also decreases gradually. With the development of the flame, the amplitudes of the disturbances of the same wavenumber increase, which indicates that with the development of the flame, the instability of the flame surface is enhanced and the disturbances with smaller wavelengths can be further developed.

Acknowledgments: The work was supported by National Natural Science Foundation of China under award 51606007, Beijing Natural Science Foundation under award 3174053, Fundamental Research Funds for the Central Universities under award 2016JBM050, and Research Foundation for Talented Scholars under award 2016 RC035.

Author Contributions: The author contributions list is as follows: Study concepts were proposed by Guo-Xiu Li. Literature research and study were designed by Guo-Xiu Li, Fu-Sheng Li, Yan-Huan Jiang, Hong-Meng Li, and Zuo-Yu Sun. Experiments studies and data acquisition were done by Fu-Sheng Li, Yan-Huan Jiang, Hong-Meng Li, and Zuo-Yu Sun. Data analysis/interpretation and statistical analysis were done by all the authors. Definition of the intellectual content of the manuscript, manuscript preparation and editing was made by Fu-Sheng Li.

Conflicts of Interest: The authors declare no conflict of interest.

\section{References}

1. Zhang, B.; Ji, C.; Wang, S. Combustion analysis and emissions characteristics of a hydrogen-blended methanol engine at various spark timings. Int. J. Hydrog. Energy 2015, 40, 4707-4716. [CrossRef]

2. Yang, K.; Zhang, H.; Wang, Z.; Zhang, J.; Yang, F.; Wang, E. Study of zeotropic mixtures of ORC (organic Rankine cycle) under engine various operating conditions. Energy 2013, 58, 494-510. [CrossRef]

3. Li, H.; Li, G.; Sun, Z.; Zhai, Y.; Zhou, Z. Measurement of the laminar burning velocities and markstein lengths of lean and stoichiometric syngas premixed flames under various hydrogen fractions. Int. J. Hydrog. Energy 2014, 39, 17371-17380. [CrossRef]

4. Ji, C.; Yang, J.; Liu, X.; Zhang, B.; Wang, S.; Gao, B. A quasi-dimensional model for combustion performance prediction of an SI hydrogen-enriched methanol engine. Int. J. Hydrog. Energy 2016, 41, 17676-17686. [CrossRef] 
5. Sun, Z.; Li, G. On reliability and flexibility of sustainable energy application route for vehicles in China. Renew. Sustain. Energy Rev. 2015, 51, 830-846. [CrossRef]

6. Kim, M.; Kim, J. Optimization model for the design and analysis of an integrated renewable hydrogen supply (IRHS) system: Application to Korea's hydrogen economy. Int. J. Hydrog. Energy 2016, 41, 16613-16626. [CrossRef]

7. Unni, J.K.; Govindappa, P.; Das, L.M. Development of hydrogen fuelled transport engine and field tests on vehicles. Int. J. Hydrog. Energy 2017, 42, 631-651.

8. Yasin, K.; İlker, G.; Tarkan, S.; Levent, Y.; Ahmet, S.D. Effect of hydrogen enrichment on combustion characteristics, emissions and performance of a diesel engine. Int. J. Hydrog. Energy 2016, 41, 656-665.

9. Yasin, K.; Illker, G.; Tarkan, S.; Levent, Y.; Ahmet, S.D.; Somchai, W. Effects of hydrogen and methane addition on combustion characteristics, emissions, and performance of a CI engine. Int. J. Hydrog. Energy 2016, 41, 1313-1325.

10. Martínez-Boggio, S.D.; Curto-Risso, P.L.; Medina, A.; Hernandez, A.C. Simulation of cycle-to-cycle variations on spark ignition engines fueled with gasoline-hydrogen blends. Int. J. Hydrog. Energy 2016, 41, 9087-9099. [CrossRef]

11. Li, H.; Li, G.; Sun, Z.; Zhou, Z.; Li, Y.; Yuan, Y. Effect of dilution on laminar burning characteristics of $\mathrm{H}_{2} / \mathrm{CO} / \mathrm{CO}_{2} /$ air premixed flames with various hydrogen fractions. Exp. Therm. Fluid Sci. 2015, 74, 160-168. [CrossRef]

12. Li, H.; Li, G.; Sun, Z.; Li, Y.; Yuan, Y. Investigation on dilution effect on laminar burning velocity of syngas premixed flames. Energy 2016, 112, 146-152. [CrossRef]

13. Lamoureux, N.; Djebaili-Chaumeix, N.; Paillard, C.E. Laminar flame velocity determination for $\mathrm{H}_{2}$-air-He- $\mathrm{CO}_{2}$ mixtures using the spherical bomb method. Exp. Therm. Fluid Sci. 2003, 27, 385-393. [CrossRef]

14. Sun, Z.; Li, G. Propagation characteristics of laminar spherical flames within homogenous hydrogen-air mixtures. Energy 2016, 116, 116-127. [CrossRef]

15. Burluka, A.A.; Gaughan, R.G.; Griffiths, J.F.; Mandilas, C.; Sheppard, C.G.W.; Woolley, R. Experimental observations on the influence of hydrogen atoms diffusion on laminar and turbulent premixed burning velocities. Fuel 2017, 189, 66-78. [CrossRef]

16. Liu, F.; Bao, X.; Gu, J.; Chen, R. Onset of cellular instabilities in spherically propagating hydrogen-air premixed laminar flames. Int. J. Hydrog. Energy 2012, 37, 11458-11465. [CrossRef]

17. Sun, Z.; Li, G.; Li, H.; Zhai, Y.; Zhou, Z. Buoyant unstable behavior of initially spherical lean hydrogen-air premixed flames. Energies 2014, 7, 4938-4956. [CrossRef]

18. Hu, E.; Huang, Z.; He, J.; Zheng, J.; Miao, H. Measurements of laminar burning velocities and onset of cellular instabilities of methane-hydrogen-air flames at elevated pressures and temperatures. Int. J. Hydrog. Energy 2009, 34, 5574-5584. [CrossRef]

19. Law, C.K.; Jomaas, G.; Bechtold, J.K. Cellular instabilities of expanding hydrogen/propane spherical flames at elevated pressures: Theory and experiment. Proc. Combust. Inst. 2005, 30, 159-167. [CrossRef]

20. Sivashinsky, G. The rate of expansion of spherical flames. Combust. Theory Model. 2006, 10, 625-637.

21. Bychkov, V.V.; Liberman, M.A. Dynamics and stability of premixed flames. Phys. Rep. 2000, 325, $115-237$. [CrossRef]

22. Yenerdag, B.; Fukushima, N.; Shimura, M.; Tanahashi, M.; Miyauchi, T. Turbulence-flame interaction and fractal characteristics of $\mathrm{H}_{2}$-air premixed flame under pressure rising condition. Proc. Combust. Inst. 2015, 35, 1277-1285. [CrossRef]

23. Mukaiyama, K.; Shibayama, S.; Kuwana, K. Fractal structures of hydrodynamically unstable and diffusive-thermally unstable flames. Combust. Flame 2013, 160, 2471-2475. [CrossRef]

24. Battista, F.; Troiani, G.; Picano, F. Fractal scaling of turbulent premixed flame fronts: Application to LES. Int. J. Heat Fluid Flow 2015, 51, 78-87. [CrossRef]

25. Hiraoka, K.; Minamoto, Y.; Shimura, M.; Naka, Y.; Fukushima, N.; Tanahashi, M. A fractal dynamic SGS combustion model for large eddy simulation of turbulent premixed flames. Combust. Sci. Technol. 2016, 188, 1472-1495. [CrossRef]

26. Ng, H.D.; Chao, J.; Yatsufusa, T.; Lee, J. Measurement and chemical kinetic prediction of detonation sensitivity and cellular structure characteristics in dimethyl ether-oxygen mixtures. Fuel 2009, 88, 124-131. [CrossRef] 
27. Diakow, P.; Cross, M.; Ciccarelli, G. Detonation characteristics of dimethyl ether and ethanol-air mixtures. Shock. Waves 2015, 25, 231-238. [CrossRef]

28. Jiang, Y.; Li, G.; Li, F.; Sun, Z.; Li, H. Experimental investigation of correlation between cellular structure of the flame front and pressure. Fuel 2017, 199, 65-75. [CrossRef]

29. Askari, O.; Elia, M.; Ferrari, M.; Metghalchi, H. Cell formation effects on the burning speeds and flame front area of synthetic gas at high pressures and temperatures. Appl. Energy 2017, 189, 568-577. [CrossRef]

30. Karlin, V.; Makhviladze, G. Computational analysis of the steady states of the Sivashinsky model of hydrodynamic flame instability. Combust. Theory Model. 2003, 7, 87-108. [CrossRef]

31. Karlin, V. Estimation of the linear transient growth of perturbations of cellular flames. Math. Models Methods Appl. Sci. 2003, 14, 1191-1210. [CrossRef]

32. Wu, J.; Wang, J.; Nie, Y.; Yu, S.; Huang, Z. Experimental study on flame instabilities of laminar premixed $\mathrm{CH}_{4} / \mathrm{H}_{2}$ /air non-adiabatic flat flames. Fuel 2015, 159, 599-606.

33. Choi, O.; Min, C.L. Investigation into the combustion instability of synthetic natural gases using high speed flame images and their proper orthogonal decomposition. Int. J. Hydrog. Energy 2016, 41, 20731-20743. [CrossRef]

34. Kawahara, N.; Tomita, E. Visualization of auto-ignition and pressure wave during knocking in a hydrogen spark-ignition engine. Int. J. Hydrog. Energy 2009, 34, 3156-3163. [CrossRef]

35. Apicella, B.; Bruno, A.; Wang, X.; Spinelli, N. Fast Fourier Transform and autocorrelation function for the analysis of complex mass spectra. Int. J. Mass Spectrom. 2013, 338, 30-38. [CrossRef]

36. Pal, B. Fourier transform ultrasound spectroscopy for the determination of wave propagation parameters. Ultrasonics 2017, 73, 140-143. [CrossRef] [PubMed]

37. Mukherjee, B.; Srivardhan, V.; Roy, P.N.S. Identification of formation interfaces by using wavelet and Fourier transforms. J. Appl. Geophys. 2016, 128, 140-149. [CrossRef]

38. Li, H.; Li, G.; Sun, Z.; Yu, S.; Zhai, Y.; Zhou, Z. Experimental investigation on laminar burning velocities and flame intrinsic instabilities of lean and stoichiometric $\mathrm{H}_{2} / \mathrm{CO} /$ air mixtures at reduced, normal and elevated pressures. Fuel 2014, 135, 279-291. [CrossRef]

39. Schoukens, J.; Rolain, Y.; Pintelon, R. Analysis of windowing/leakage effects in frequency response function measurements. Automatica 2006, 42, 27-38. [CrossRef]

40. Belega, D.; Petri, D.; Dallet, D. Impact of harmonics on the interpolated DFT frequency estimator. Mech. Syst. Signal Process. 2015, 66, 349-360. [CrossRef]

41. Tabb, D.L.; Shah, M.B.; Strader, M.B.; Conelly, H.M.; Hettich, R.L.; Hurst, G.B. Determination of peptide and protein ion charge states by Fourier transformation of isotope-resolved mass spectra. J. Am. Soc. Mass Spectrom. 2006, 17, 903-915. [CrossRef] [PubMed] 